Revista de Investigación Educativa 25

julio-diciembre, 2017 | ISSN 1870-5308 | Xalapa, Veracruz

Instituto de Investigaciones en Educación | Universidad Veracruzana

\title{
Desarrollo de la capacidad de memoria semántica a través de la formación de redes relacionadas en estudiantes con trastorno del espectro autista
}

\section{Development of semantic memory ability through the training of related networks in students with autism spectrum disorder}

\author{
Dr. Manuel Ojea Rúa \\ Profesor \\ Universidad de Vigo, España \\ moxea@uvigo.es
}

Este estudio presenta el análisis de la efectividad de un programa, cuya finalidad es facilitar el desarrollo de la memoria semántica y sus relaciones en estudiantes con trastorno del espectro autista, diseñado bajo los principios teóricos de la teoría de la percepción y la teoría de la coherencia central cognitiva. Para llevarlo a cabo, se ha utilizado un diseño experimental pretests-programa-postests de dos grupos, uno experimental y otro de control, en el cual han participado 14 estudiantes de 13 años de edad, distribuidos equitativamente entre ambos grupos. Los resultados muestran que el programa responde positivamente a los objetivos de la investigación, ya que los estudiantes que integran el grupo experimental han mejorado significativamente en relación con sus pares del grupo control.

Palabras clave: Percepción; autismo; cognición; memoria.

Study presents the analysis of the efficacy of a program to issue the development of semantic memory and relationships in students with autism spectrum disorder, designed the theoretical principles of the theory of perception and theory of central cognitive coherence. To do this study, a pre-tests-program-pos-tests experimental design of two groups, an experimental group and a control group, in which 14 students participate, of 13 years, equally distributed between both groups. The results show that the program responds positively to the objectives of the research, since the students the experimental group has improved the significantly compared with the pairs of the control group.

Key words: Perception; autism; cognition; memory. 


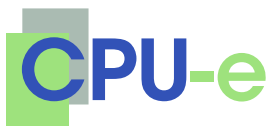

Desarrollo de la capacidad de memoria semántica a

través de la formación de redes relacionadas

Manuel Ojea Rúa

\title{
Desarrollo de la capacidad de memoria semántica a través de la formación de redes relacionadas en estudiantes con trastorno del espectro autista
}

\author{
Development of semantic memory ability through the training of \\ related networks in students with autism spectrum disorder
}

\section{Introducción}

$\mathrm{D}$ e acuerdo con la Clasificación Internacional de las Discapacidades (American Psychiatric Association, 2013), el desarrollo psicosocial y educativo de las personas con trastorno del espectro autista (en adelante TEA), en sus tres niveles del continuo del desarrollo, se caracterizan por déficits nucleares en el ámbito de la interacción y la comunicación social. Las teorías del procesamiento de la información y las teorías del aprendizaje social aportan explicaciones cognitivas válidas acerca de la presencia de dichas limitaciones, las cuales son debidas, entre otras causas, a dificultades estructurales relacionadas con la capacidad o competencia de integración de la información en su plano semántico y procedimental (Belinchón, Rivière \& Igoa, 1992).

Esta capacidad de integración conceptual-categorial depende, fundamentalmente, de la percepción global o gestalt de los estímulos entrantes o procesos de aprendizaje (Bölte, Holtman, Poustka, Scheurich \& Schmidt, 2007; Frith, 2004; Rondan \& Deruelle, 2007). Ahora bien, la adquisición del procesamiento cognitivo en las personas con TEA en términos de integración de la totalidad o de contenidos semánticos, depende, a su vez, de la construcción progresiva (sucesiva) de las partes y, para que esto sea posible, han de establecerse adecuadamente los procesos de relación interactivos (mediación cognitiva) entre los nuevos aprendizajes (estímulos externos) y aquellos aprendizajes previamente adquiridos y debidamente codificados (estímulos internos). Dicho desarrollo secuencial precisa la articulación de una estructura sistemática de los procesos funcionales de mediación cognitiva instrumental (nexosrelaciones), lo que Vygotsky (1972; 1979) denomina método instrumental, el cual indi- 
ca el modo en que usan los mediadores que, sin alterar la tarea, permiten facilitar la adquisición de los estímulos, lo que mejora sensiblemente la representación cognitiva de éstos y, por consiguiente, la ejecución de la tarea y el desarrollo de las operaciones mentales relacionadas.

Efectivamente, son numerosos los estudios que inciden en estas líneas de investigación. Walsh, Creighton y Rutherford (2016) concluyen que los déficits en el procesamiento de la información en las personas con TEA son los responsables de las limitaciones en la percepción emocional y en tareas cognitivas complejas de tipo cognitivo social. Maister y Plaisted-Grant (2011) implican, asimismo, a dificultades en el desarrollo de los procesos temporales, así como a errores en la reproducción de acontecimientos ordenados en el tiempo, con limitaciones evidentes en la ejecución de tareas cognitivas sociales (comprensión social) y de memoria episódica en personas con TEA. Evers, de Wit, Van der Hallen, Haesen, Steyaert, Noens y Wagemans (2014) afirman que los déficits perceptivos hallados en las personas con TEA se relacionan con limitaciones en la capacidad de integración perceptiva de los estímulos cuando es necesario rastrearlos o distinguirlos en movimiento, debido, entre otras causas, a los déficits hallados en los procesos perceptivos, relacionados con la atención conjunta. Hamilton, Brindley y Frith (2009) comprueban, mediante los estudios basados en neuroimagen, realizados en base a tareas de rendimiento en actividades de análisis de la perspectiva visual, que cuando éstas se relacionan con tareas de rotación mental, las personas con TEA presentan limitaciones severas en tareas que implican niveles relacionales cognitivos.

La percepción explica que todo lo que conocemos y aprendemos acerca del medio que nos rodea y de nosotros mismos tiene su origen en nuestros sentidos, mediante el proceso de recogida, análisis, interpretación y comprensión de la información que recibimos constantemente. A la información recibida hemos de atribuir un sentido o significado en términos de globalidad (semántica) para poder entender el contenido informativo y relacionarlo consiguientemente con aquella información disponible con anterioridad en la memoria permanente.

Es precisamente en este nivel de codificación semántica donde las personas con TEA presentan sus mayores limitaciones. Yamasaki, Fijita, Ogata, Goto, Munetsuna, Kamio y Tobimatsu (2011) afirman que las personas con TEA presentan evidencias de limitaciones en déficits de atribución perceptiva global, con una tendencia a centrarse en los detalles de los estímulos percibidos. Sus estudios, basados en el uso de potenciales evocados, evidencian electrofisiológicamente la presencia de estas limitaciones en la atribución de significados o contenidos semánticos. Sheppard, Ropar, 
Underwood y van Loon (2010) hallan, asimismo, que las personas con TEA presentan mayores dificultades para integrar los peligros en tareas de conducción cuando el análisis se realiza de forma global; sin embargo, cuando el peligro de la tarea se relaciona directamente con las consecuencias de una persona concreta, las personas con TEA no presentan diferencias en relación con el proceso perceptivo de las personas normotípicas. Iarocci, Rombough, Yager, Weeks y Chua (2010) confirman que las personas con TEA presentan déficits en los procesos de ejecución de actividades globalizadas relacionadas con tareas de lectura labial, ya que centran la percepción del estímulo en sus aspectos parciales, sin que se observen interferencias importantes del proceso bimodal (gestos de los labios). Solières, Mottron, Saumier y Larochelle (2007) observan que las personas con TEA presentan una menor capacidad de discriminación en la adquisición de conceptos y codificación de categorías o agrupación jerarquizada de conceptos (globalidad), debido, sobre todo, a limitaciones para el desarrollo de relaciones entre el conocimiento categorizado.

Se evidencia, así, que la percepción depende del aprendizaje, pero también, va a depender de la madurez cognitiva, la cual influye decisivamente en los procesos de codificación e interpretación del aprendizaje recibido; se trata de un proceso ampliamente relacionado, en el cual la coordinación entre las entradas y las salidas de la información constituye una condición básica para un adecuado funcionamiento del sistema cognitivo. En efecto, el mundo real percibido y el mundo que se percibe desde la óptica del perceptor son sensiblemente diferentes, lo cual es debido a las diferencias existentes entre la actividad manifiesta de la recepción estimular y las interpretaciones personales realizadas sobre éstas. Las respuestas a los sistemas sensoriales se expresan en el proceso de codificación gracias al mecanismo conceptual de transducción funcional y a las modulaciones que pueden afectar a la transmisión del impulso neuronal. Según este mecanismo cognitivo, para que esta codificación tenga efectos perceptivos, es preciso que los eventos neuronales constituyan símbolos o representaciones consideradas relevantes de la estimulación percibida, de forma que el sistema cognitivo atribuya a las características de las transacciones físicas la relevancia que facilite la priorización de dicho contenido, delimitando cuáles serán las transacciones que han de ser seleccionadas y cuáles podrán ser rechazadas, así como el objeto de tratamiento que tendrán dichos datos, tanto si se trata de una representación como de una percepción simbólica. La percepción es, entonces, un proceso activo, el cual modifica un tipo de representación cognitiva en otro, a través de una o más operaciones de procesamiento, con la única especificidad de que su punto de partida son los 
datos sensoriales y las operaciones iniciales que están guiadas por esos mismos datos (Dretske, 1981; Gardner, 1985; Fodor, 1983; Liu, Cherkassky, Minshew \& Just, 2011; Rumelhart \& McClelland, 1986).

Bajo esta delimitación conceptual, la percepción conforma la interacción de ambas etapas de la actividad perceptiva, la guiada por los datos y la guiada cognitivamente; ahora bien, cada una de estas etapas posee límites y restricciones, ya que ambos tipos perceptivos intervienen en diferentes momentos y con distintos logros cognitivos. Los primeros, esto es, la percepción guiada por los datos, posee un carácter relativamente superficial, alejado de los niveles superiores cognitivos, los cuales se encargan del análisis perceptivo y se caracterizan por su rapidez en el procesamiento, mientras que los segundos, esto es, la percepción guiada cognitivamente, son ya los encargados de la fijación de las creencias perceptivas, que es donde las representaciones distales (que constituyen las propiedades de los eventos percibidos) interactúan con los ouputs de otros módulos cognitivos. Posteriormente, los contenidos pasan a formar parte de los sistemas centrales y se vuelven disponibles en la memoria de trabajo para ser utilizados en procesos subsiguientes de razonamiento, planificación y ejecución. Durante este proceso, las áreas de proyección cortical desempeñan un papel fundamental, ya que, por un lado, facilitan la integración de los impulsos que proceden de un mismo conjunto de receptores y, por otro, permiten la interacción de unas áreas con otras, posibilitando la comunicación entre modalidades sensoriales diferentes y la formación de agrupaciones neuronales de rango superior. De este modo, la percepción va más allá de la simple actividad sensorial, en la cual las personas operan con representaciones cognitivas, que van transformando la información y la transcienden, con el fin de llegar a una representación estable y consistente del mundo observado (Gibson, 1979; Johnson-Laird, 1983; Pylyshyn, 1984).

Los estudios señalados muestran que las personas con TEA manifiestan una alteración perceptiva en la evaluación de la experiencia, debido, entre otros motivos, a dificultades importantes en la complementariedad o desarrollo de relaciones entre ambos niveles de información perceptual, el sistema perceptivo de abajo-arriba o guiado por los datos, y el sistema guiado cognitivamente o de arriba-abajo. Estas limitaciones están determinadas por un trastorno de la incorporación estimular, consistente en una extrañeza de los objetos en su relación interactiva, en la cual, las personas con TEA viven el mundo como novedoso cada vez, caracterizado por una gran distancia emocional, que concluye con los criterios de diagnóstico relacionados con déficits evidentes en tareas de comprensión social (Ojea, 2006, 2008a). 
Siguiendo los presupuestos teóricos indicados, este estudio tiene como finalidades principales, en primer lugar, ofrecer una respuesta integrada a las limitaciones existentes en los niveles del procesamiento de la información en los estudiantes con TEA, en relación con los contenidos adquiridos de memoria semántica. Para lograr este objetivo básico se ha llevado a cabo el diseño de un programa específico facilitador de la formación de relaciones significativas, primero, entre los contenidos entrantes con aquellos otros previamente aprendidos y, segundo, entre los contenidos aprendidos entre sí. Para lo cual, el programa se apoya en el uso de claves o relaciones interconceptuales, cuyo objetivo último es permitir el desarrollo de la capacidad de memoria semántica en las personas con TEA.

\section{Objetivos}

Con estas premisas básicas, en este estudio se pretende, pues, responder a los siguientes objetivos generales:

1. Diseñar un programa específico para facilitar el desarrollo de la memoria semántica, mediante el establecimiento de relaciones nodales en estudiantes con TEA.

2. Aplicar dicho programa específico a los estudiantes con TEA, que integran un grupo experimental, con la finalidad de comparar su efectividad, en relación con sus pares de un grupo control, quienes han continuado con las actividades académicas ordinarias.

3. Analizar el desarrollo progresivo de la aplicación del programa, considerando las medidas de las variables: memoria semántica y rendimiento, en ambos grupos del estudio.

\section{Método}

\subsection{Diseño}

Este estudio se ajusta a un diseño experimental pretests-programa-postests, con medidas repetidas, para dos grupos, un grupo experimental (GE), formado por 7 estudiantes con TEA, y un grupo control (GC), formado, asimismo, por otros 7 estudiantes 
con TEA, distribuidos entre ambos grupos de forma equitativa en función de sus características personales.

El procedimiento de selección de la muestra se ha apoyado en un proceso aleatorio entre los estudiantes que cumplen, entre otras, las siguientes condiciones básicas: 1) tener un diagnóstico oficial previo de TEA; 2) realizar estudios de educación secundaria, y 3 ) recibir atención específica en asociaciones o servicios específicos de apoyo de forma interna o externa al centro educativo.

Asimismo, se han solicitado las autorizaciones correspondientes a las familias de los estudiantes que han participado en el estudio.

\subsection{Participantes}

En el estudio han participado 14 estudiantes, con diagnóstico de TEA de nivel 1, con diagnóstico específico previo emitido por los servicios educativos correspondientes, de acuerdo con los criterios de diagnóstico establecidos por la American Psychiatric Association (2013), de edades comprendidas entre los 13 y 14 años de edad, que cursan $2^{\circ}$ curso de educación secundaria obligatoria en diferentes centros educativos de la provincia de Ourense (España); algunos combinan su atención psicoeducativa en una Asociación específica de atención al alumnado con trastornos de la comunicación social; 12 son hombres y dos son mujeres; dos presentan nivel cognitivo mediobajo, 10 nivel cognitivo medio y dos nivel cognitivo medio-alto.

Como puede observarse en la Tabla 1, los participantes han sido distribuidos, pues, en dos grupos, un grupo experimental y un grupo control.

Tabla 1. Distribución de los participantes

\begin{tabular}{|c|c|c|c|c|c|c|}
\hline & \multirow{2}{*}{\multicolumn{6}{|c|}{ Sexo }} \\
\hline & & & & & & \\
\hline & \multicolumn{3}{|c|}{ Hombre } & \multicolumn{3}{|c|}{ Mujer } \\
\hline & \multicolumn{6}{|c|}{ Nivel cognitivo } \\
\hline & Medio-bajo & Medio & Medio-alto & Medio-bajo & Medio & Medio-alto \\
\hline $\begin{array}{l}\text { Grupo } \\
\text { Experimental (GE) }\end{array}$ & 1 & 4 & 1 & & 1 & \\
\hline Grupo Control (GC) & 1 & 4 & 1 & & 1 & \\
\hline Total & 2 & 8 & 2 & & 2 & \\
\hline
\end{tabular}

Fuente. Elaboración propia. 


\subsection{Variables}

Las variables sistematizadas para este estudio son las siguientes:

1. La capacidad de memoria semántica: MY, nivel III, evaluada en puntuaciones percentiles ( $\mathrm{pc}$ ) (o-10o), a lo largo de tres medidas repetidas $\left(\mathrm{MY}_{1-2-3}\right)$.

2. El rendimiento académico: $\mathrm{RA}_{1-2}$, con dos valores de medidas repetidas, antes y después de la aplicación del programa, expresado, asimismo, en puntuaciones porcentuales $(\mathrm{pc})$.

\subsection{Instrumentos}

Las puntuaciones referidas a las variables indicadas han sido halladas a partir de los siguientes instrumentos de medida: 1) las puntuaciones de la variable memoria semántica (MY), nivel III, han sido obtenidas a través del test MY de Memoria-III de Yuste (2005) (ver Anexo 1), y 2) los datos de la variable rendimiento académico (RA) han sido hallados a partir de los resultados académicos indicados en la evaluación curricular de los estudiantes.

\subsection{Procedimiento}

El estudio se ha desarrollado a lo largo de un curso escolar (10 meses). Los participantes del GE han recibido una sesión semanal, de tipo individual, con el fin de facilitar la formación de nodos entre los contenidos trabajados en sus correspondientes grupos. Por su parte, los integrantes del GC no han recibido estas sesiones y continuaron sus actividades curriculares regulares.

De manera previa al inicio de las actividades, se ha procedido a realizar una primera medida referida a los valores de las variables $\mathrm{MY}_{1} \mathrm{Y} \mathrm{RA}_{1}$ de todos los participantes en el estudio. Posteriormente, se llevó a cabo el diseño del marco programático de referencia, el cual ha sido aplicado a los participantes que conforman el GE, mientras que los participantes incluidos en el GC continuaron con sus actividades ordinarias, previstas en los programas regulares y/o de refuerzo del centro educativo. Finalmente, con el fin de comparar las intervenciones realizadas entre ambos grupos, se han recogido otras dos medidas en la variable $\mathrm{MY}_{2-3}$, así como una segunda medida en la variable $\mathrm{RA}_{2}$. 


\section{El programa}

El proceso programático diseñado para su aplicación en el GE del estudio se ha estructurado a partir de los presupuestos teóricos expuestos, y está conformado por tres dimensiones generales: I. Integración sensorial sucesiva, II. Construcción gestáltica, y III. Memoria semántica.

I. Integración sensorial sucesiva

1.1. Presentación del estímulo global.

1.2. Subdivisión del estímulo en las partes que lo componen, de acuerdo con la atribución conceptual previamente evaluada.

1.3. Construcción de contenidos mediadores (R).

1.4. Recomposición del estímulo inicial, uniendo las partes de forma sucesiva, debidamente relacionadas con los contenidos mediadores.

\section{Construcción gestáltica}

2.1. Codificación estratégica del estímulo recompuesto: concepto.

2.2. Análisis conceptual de los mediadores instrumentales (R).

2.3. Resolución: contenido.

2.4. Jerarquización del proceso conceptual: categorías.

\section{III.Memoria semántica}

3.1. Recuperación del mediador relacionado (R).

3.2. Recuperación de la información.

3.3. Aplicación del contenido.

3.4. Generalización del contenido.

A modo de ejemplo, se expone un extracto del programa. Así, en la actividad de matemáticas de $2^{\circ}$ de educación secundaria obligatoria, correspondiente al tema de la proporcionalidad numérica, se diseña la siguiente secuencia de aprendizaje:

I. Integración sensorial sucesiva

1.1. Estímulo global: "Lis duerme 8 horas diarias, ¿cuál es la razón entre el tiempo que duerme y el total?"

1.2. Subdivisión: "[Lis duerme 8 horas al día] [El día completo tiene 24 horas] [La razón es la proporción de las horas que duerme Lis en relación a las horas totales que tiene 1 día] [Para hallar la proporción se divide el número de horas dormidas por el total de horas del día, multiplicadas por 100]”.

1.3. "[Lis duerme 8 horas al día]-R-(fases del giro del sol que se corresponde con 1 día completo: mañana-mediodía-tarde-noche)-R-(en clase somos 
22 estudiantes: $100 \%$, hoy faltan 2) [El día completo tiene 24 horas]"-R(temporalizo las actividades que hago a lo largo del día en mi agenda) "[La razón es la proporción de las horas que duerme Lis en relación a las horas totales que tiene 1 día]-R-(observo la hora en que me acuesto: las 11:0o horas, observo la hora en que me levanto: 7:0o horas, cuántas horas he dormido: 8:0o horas)".

1.4. "Lis duerme 8 horas diarias, ¿cuál es la razón entre el tiempo que duerme y el total?"-La proporción relativa a las horas que Lis duerme: 8 , en relación al total de horas que tiene 1 día: 24 .

II. Construcción gestáltica

2.1. "[Para hallar la proporción se divide el número de horas dormidas por el total de horas del día, multiplicadas por 100] [8/24: 0.33] [0.33*100: $33.3 \%]$ ”.

2.2. Análisis conceptual de los mediadores $(\mathbf{R})$ :

$\mathbf{R}$ (fases del giro del sol que se corresponde con 1 día completo: mañana-mediodía-tarde-noche): -Hago fotografías de la puesta del sol, de la salida del sol. $\mathbf{R}$ (observo la hora a la que me acuesto: las 11:00 horas: observo la hora a la que me levanto: 7:00 horas; cuántas horas he dormido: 8:00 horas): -pongo el despertador al acostarme, detengo el despertador cuando suena al despertarme-sumo las horas que he dormido-divido las horas que he dormido por el número total de horas que tiene el día.

$\mathbf{R}$ (en clase somos 22 estudiantes: $100 \%$, hoy faltan 2): -Los 2 estudiantes que faltan se llaman (...)-los 22 estudiantes suponen el 100\%-al faltar 2 estudiantes, entonces divido 2/22: 0.09-multiplico 0.09*100: 9.09: porcentaje de los que faltan: $9.09 \%$.

$\mathbf{R}$ (temporalizo las actividades que hago a lo largo del día en mi agenda): -Escribo en mi agenda las actividades principales que hago a lo largo del día, indicando la hora correspondiente-escribo un pequeño diario de las actividades que hago en las diferentes horas del día.

2.3. Resolución: contenido: [8/24: 0.33 ] [0.33*100: $33.3 \%]$.

2.4. " 8 horas cada día son 56 horas en una semana ( 7 días), una semana tiene 168 horas, por tanto, duermo 56/168: 0.33*100: 33.3\%” (...)

III. Memoria semántica

3.1. Mediador (R):

(Fases del giro del sol que se corresponde con 1 día completo: mañanamediodía-tarde-noche): un día completo: 24 horas. 
(Observo la hora a la que me acuesto: las 11:0o horas, observo la hora a la que me levanto: 7:0o horas): 8 horas.

(...)

3.2. Relación del mediador $(R)$ con el concepto:

24 horas: $100 \%$.

8 horas: $8 / 24: 0.33^{*} 100: 33.3 \%$.

Proporción (Razón): 33.3\%.

3.3. Aplicación:

"Dedico 3 horas al estudio al día-3/24: 0.125*100: 12.5\% del día” (...)

Otras tareas (...)

3.4. Generalización:

"En mi centro, hay 400 estudiantes, de los cuales, 210 son niñas, ¿qué porcentaje hay de niños y niñas?”

"Tenía 20 euros, he gastado esta semana 10 euros, ¿qué porcentaje de dinero he gastado?” (...)

\section{Resultados}

\subsection{Estudio de la distribución muestral}

Con la finalidad de comparar los datos obtenidos, en relación con aquellos que cabría esperar a priori en este estudio, se ha procedido a realizar la prueba no paramétrica Chi Cuadrado (Tabla 2).

\section{Tabla 2. Estadísticos de contraste}

\begin{tabular}{cccccc}
\hline & $\mathbf{M Y}_{\mathbf{1}}$ & $\mathbf{M Y}_{\mathbf{2}}$ & $\mathbf{M Y}_{\mathbf{3}}$ & $\mathbf{R A}_{\mathbf{1}}$ & $\mathbf{R A}_{\mathbf{2}}$ \\
\hline Chi-cuadrado & 1.14 & 8.85 & 1.00 & 3.85 & 3.14 \\
\hline Gl & 1 & 4 & 4 & 4 & 5 \\
\hline Sig. asintót. & .28 & .06 & .91 & .42 & .67 \\
\hline
\end{tabular}

Fuente: Elaboración propia. 
Los resultados de dicha prueba permiten deducir, de acuerdo con los niveles críticos indicados, que la distribución muestral se ajusta a la uniformidad, encontrándose altos valores diferenciales entre los recuentos observados y los esperados, lo que indica una alta relación entre las variables y una alta probabilidad de aleatoriedad de la muestra.

En lo que refiere a los niveles de la normalidad de la muestra, cuyos análisis se han realizado mediante la prueba no paramétrica de Kolmogorov-Smirnov, se observa, efectivamente, que sólo para la variable $\mathrm{MY}_{1}$, para un $p$-valor de .05, se obtienen datos del nivel de significación (.02), indicativos de una distribución que no se ajusta a la normalidad, mientras que los resultados obtenidos para las restantes variables señalan niveles críticos, que permiten concluir que la distribución del estudio se ajusta a la normalidad muestral (Tabla 3 ).

Tabla 3. Prueba de Kolmogorov-Smirnov para una muestra

\begin{tabular}{ccccccc}
\hline & & $\mathbf{M Y}_{\mathbf{1}}$ & $\mathbf{M Y}_{\mathbf{2}}$ & $\mathbf{M Y}_{\mathbf{3}}$ & $\mathbf{R A}_{\mathbf{1}}$ & $\mathbf{R A}_{\mathbf{2}}$ \\
\hline \multirow{3}{*}{ Parámetros normales $^{\mathbf{a}, \mathbf{b}}$} & Media & 1.64 & 2.64 & 3.92 & 4.07 & 5.64 \\
\cline { 2 - 7 } & $\begin{array}{c}\text { Desviación } \\
\text { típica }\end{array}$ & .49 & 1.08 & 1.439 & 1.20 & 1.86 \\
\hline \multirow{3}{*}{ Diferencias más extremas } & Absoluta & .40 & .29 & .20 & .20 & .21 \\
\cline { 2 - 7 } & Positiva & .25 & .29 & .16 & .14 & .21 \\
\cline { 2 - 7 } & Negativa & -.40 & -.20 & -.20 & -.20 & -.11 \\
\hline Z de Kolmogorov-Smirnov & & 1.52 & 1.10 & .74 & .77 & .78 \\
\hline Sig. asintót. (bilateral) & & .02 & .17 & .62 & .58 & .56 \\
\hline
\end{tabular}

Fuente: Elaboración propia.

Por otra parte, las observaciones halladas se ajustan a una distribución aleatoria de las frecuencias. En dicho análisis, realizado mediante la prueba no paramétrica de Rachas, se concluye, pues, que la muestra se ajusta a una distribución caracterizada por la aleatoriedad de las frecuencias observadas en todas las variables del análisis, tal como se indica en los niveles críticos alcanzados (Tabla 4). 
Tabla 4. Prueba de Rachas

\begin{tabular}{lccccc}
\hline & $\mathbf{M Y}_{\mathbf{1}}$ & $\mathbf{M Y}_{\mathbf{2}}$ & $\mathbf{M Y}_{\mathbf{3}}$ & $\mathbf{R A}_{\mathbf{1}}$ & $\mathbf{R A}_{\mathbf{2}}$ \\
\hline Valor de prueba & $\mathbf{2}$ & 2.00 & 4.00 & 4.00 & 5.00 \\
\hline Casos $<$ Valor de prueba & 5 & 1 & 6 & 4 & 4 \\
\hline Casos >= Valor de prueba & 9 & 13 & 8 & 10 & 10 \\
\hline Casos en total & 14 & 14 & 14 & 14 & 14 \\
\hline Número de rachas & 10 & 3 & 4 & 5 & 6 \\
\hline Z & 1.26 & .00 & -1.91 & -.84 & -.14 \\
\hline Sig. asintót. (bilateral) & .20 & 1.00 & .05 & .39 & .88 \\
\hline
\end{tabular}

a Mediana.

Fuente: Elaboración propia.

\subsection{Análisis estadístico preliminar}

Los análisis de los datos obtenidos en las medidas repetidas, realizados para ambas variables: MY y RA, tanto en relación a los participantes del GE, como de los que conforman el GC, se corresponden con los estadísticos generales presentados en la Tabla 5.

Tabla 5. Estadísticos descriptivos (N: 14)

\begin{tabular}{ccccccc}
\hline & Suma & Media & $\begin{array}{c}\text { Desviación } \\
\text { típica }\end{array}$ & Mínimo & Máximo & Varianza \\
\hline $\mathrm{MY}$ & 23.00 & 1.64 & .49 & 1.00 & 2.00 & .25 \\
\hline $\mathrm{MY}_{2}$ & 37.00 & 2.64 & 1.08 & 1.00 & 5.00 & 1.17 \\
\hline $\mathrm{MY}_{3}$ & 55.00 & 3.92 & 1.43 & 2.00 & 6.00 & 2.07 \\
\hline $\mathrm{RA}_{1}$ & 57.00 & 4.07 & 1.20 & 2.00 & 6.00 & 1.46 \\
\hline $\mathrm{RA}_{2}$ & 79.00 & 5.64 & 1.86 & 3.00 & 9.00 & 3.48 \\
\hline
\end{tabular}

Fuente: Elaboración propia.

Es destacable que en los datos referidos a la suma de las puntuaciones se observa un incremento progresivo, tanto en las medidas repetidas de la variable MY, como en las medidas pre-post de la variable RA; en $\mathrm{MY}_{1}$ es de 23 y en $\mathrm{MY}_{3}$, de 55 , mientras que en $\mathrm{RA}_{1}$ es de $57 \mathrm{y}$ en $\mathrm{RA}_{3}$, de 79.0o. Por otra parte, el estadístico de la media de las puntuaciones se incrementa, asimismo, sensiblemente, a lo largo de las medidas sucesivas 
del estudio; en la variable $\mathrm{MY}_{1}$ la puntuación media es de 1.64, mientras que en $\mathrm{MY}_{3}$ se obtiene una media de 3.92. Del mismo modo, en la variable RA, la puntuación media se incrementa desde $\mathrm{RA}_{1}: 4.07$ hasta 5.64 en $\mathrm{RA}_{2}$.

La línea ascendente de las puntuaciones se relaciona con las mejoras halladas en los datos referidos a todos los estudiantes; la cuestión es, entonces, analizar si existen diferencias significativas en los participantes que conforman el GE en relación con sus homólogos del GC.

Pues bien, en la Tabla 6, pueden observarse los rangos de las puntuaciones promedio obtenidas, según la pertenencia de los participantes al tipo grupo asignado: GE y GC.

\section{Tabla 6. Rangos promedio, según el tipo de grupo}

\begin{tabular}{cccc}
\hline & Grupo & N & Rango promedio \\
\hline \multirow{2}{*}{$M Y_{1}$} & $\mathrm{GE}$ & 7 & 8.00 \\
\cline { 2 - 4 } & $\mathrm{GC}$ & 7 & 7.00 \\
\hline \multirow{2}{*}{$\mathrm{MY}_{2}$} & $\mathrm{GE}$ & 7 & 9.86 \\
\cline { 2 - 4 } & $\mathrm{GC}$ & 7 & 5.14 \\
\hline \multirow{2}{*}{$\mathrm{MY}_{3}$} & $\mathrm{GE}$ & 7 & 10.93 \\
\cline { 2 - 4 } & $\mathrm{GC}$ & 7 & 4.07 \\
\hline \multirow{2}{*}{$\mathrm{RA}_{1}$} & $\mathrm{GE}$ & 7 & 7.64 \\
\cline { 2 - 4 } & $\mathrm{GC}$ & 7 & 7.36 \\
\hline \multirow{2}{*}{$\mathrm{RA}_{2}$} & $\mathrm{GE}$ & 7 & 9.79 \\
\cline { 2 - 4 } & $\mathrm{GC}$ & 7 & 5.21 \\
\hline
\end{tabular}

Fuente: Elaboración propia.

\subsection{Análisis comparativo relacionado}

De esta forma, puede concluirse que los participantes que integran el GE incrementan el rango promedio de las puntuaciones en las medidas sucesivas, tanto en MY como en RA. Así, pasan de una puntuación de 8.00 en $\mathrm{MY}_{1}$ a 10.93 en $\mathrm{MY}_{3}$; en RA aumentan el rango promedio desde $\mathrm{RA}_{1}: 7.64$ a 9.79 en $\mathrm{RA}_{2}$. Sin embargo, no se produce esta misma secuencia en los participantes del GC, quienes disminuyen progresivamente los rangos promedio, así, de 7.00 en $\mathrm{MY}_{1}$ pasan a 4.97 en $\mathrm{MY}_{3}$, y de una puntuación de 7.36 en $\mathrm{RA}_{1}$ pasan a obtener una puntuación de 5.21 en $\mathrm{RA}_{2}$. 
Pues bien, consideradas estas puntuaciones sucesivas halladas como $k$ muestras relacionadas, los cálculos realizados mediante la prueba de Friedman permiten concluir que existen, en efecto, diferencias significativas entre las puntuaciones sucesivas, tanto en MY como en RA, para el conjunto del estudio ( $\mathrm{N}: 14$ ), en el cual se ha obtenido un nivel crítico de significación de .oo (Chi cuadrado: 45.03, con 4 grados de libertad), con los rangos promedio (Tabla 7 ).

\section{Tabla 7. Prueba comparativa de Friedman}

\begin{tabular}{|c|c|}
\hline $\mathrm{N}$ & 14 \\
\hline Chi-cuadrado & 45.035 \\
\hline $\mathrm{Gl}$ & 4 \\
\hline Sig. asintót.a & .000 \\
\hline
\end{tabular}

a Contraste de Friedman.

Fuente: Elaboración propia.

\subsection{Análisis comparativo en relación con la variable "grupo"}

Ahora bien, para comparar los niveles diferenciales de los cambios hallados en las diferentes variables del estudio, en relación con la variable tipo de grupo (grupo de participantes: GE y GC), se llevan a cabo las medidas sucesivas considerando las variables como muestras independientes, en relación con el tipo grupo de participantes, las cuales han sido obtenidas mediante la prueba no paramétrica para $k$ muestras independientes de Kruskall-Wallis (Tabla 8).

Tabla 8. Estadísticos de contraste $(a, b)$

\begin{tabular}{lccccc}
\hline & $\mathbf{M Y}$ & $\mathbf{M Y}_{\mathbf{2}}$ & $\mathbf{M Y}_{\mathbf{3}}$ & $\mathbf{R A}_{\mathbf{1}}$ & $\mathbf{R A}_{\mathbf{2}}$ \\
\hline Chi-cuadrado & .28 & 5.13 & 9.83 & .01 & 4.36 \\
\hline Gl & 1 & 1 & 1 & 1 & 1 \\
\hline Sig. asintót. & .59 & .02 & .00 & .89 & .03 \\
\hline
\end{tabular}

a) Prueba de Kruskal-Wallis.

b) Variable de agrupación: GRUPO.

Fuente: Elaboración propia. 
Pues bien, los datos hallados permiten, entre otras, realizar las siguientes conclusiones:

1. En las puntuaciones de la primera medida de la variable $\mathbf{M Y}_{1}$ no se hallan diferencias significativas entre ambos grupos (sig: .59).

2. En las medidas sucesivas de $\mathrm{MY}_{2-3}$ las diferencias, sin embargo, alcanzan niveles de significatividad cada vez mayores entre los participantes del GE y del GC (sig: .02 y .oo en $\mathrm{MY}_{1}$ y $\mathrm{MY}_{2}$ respectivamente).

3. En la variable $\mathrm{RA}_{1}$ no existen diferencias significativas en las puntuaciones obtenidas entre los participantes de ambos grupos: GE y GC (sig: .89).

4. En la variable $\mathrm{RA}_{2}$ se aprecian, no obstante, diferencias en las puntuaciones entre ambos grupos (sig: .03).

En conclusión, en la medida que avanza el desarrollo educativo propuesto, las diferencias en función del tipo de grupo se hacen cada vez más evidentes entre el GE y sus pares del GC.

En efecto, en las Figuras 1 y 2, pueden observarse visualmente las diferencias en el proceso de evolución en las medidas repetidas de la variable MY, así como de la variable RA, agrupadas en relación con el tipo de grupo: GE y GC.

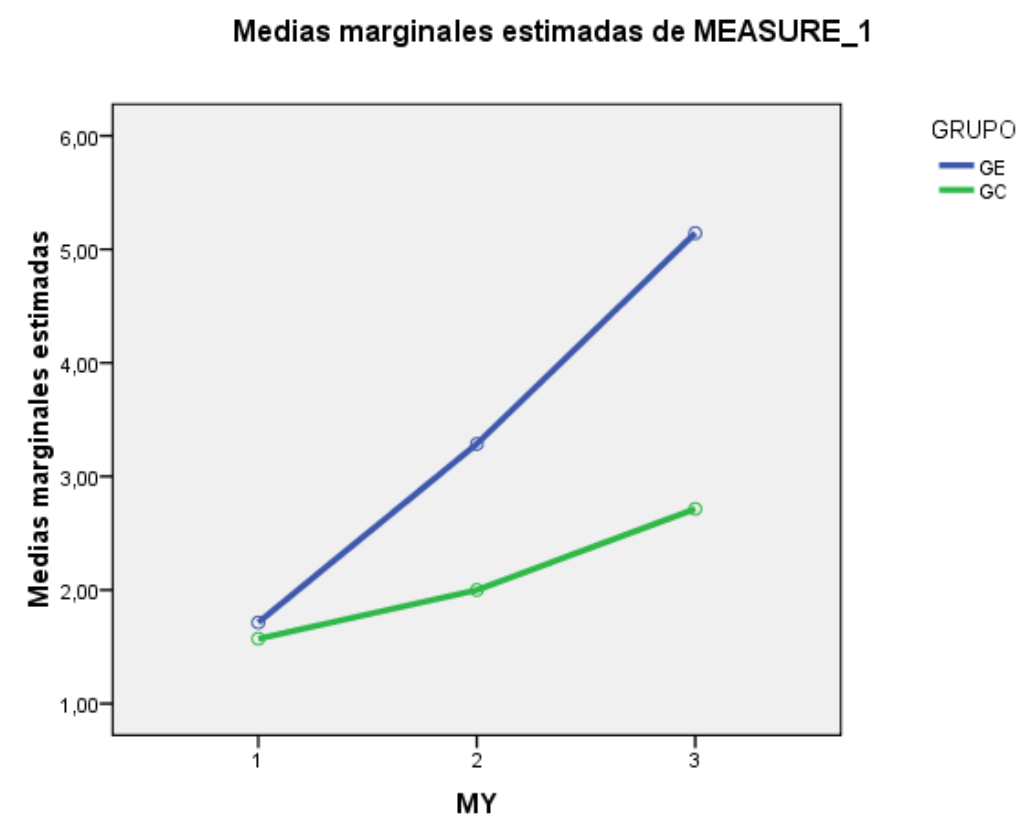

Figura 1. Evolución en la variable MY, según el tipo de grupo 
Como se observa en la Figura 1, la línea indicativa de los datos hallados en la variable MY señala una curva ascendente claramente superior en los estudiantes que conforman el GE, en las tres medidas repetidas, en relación con sus homólogos del GC.

Asimismo, en la Figura 2, se aprecia un trazado similar al anterior para las dos medidas repetidas de la variable RA, cuyos datos son sensiblemente superiores en los estudiantes que conforman el GE, en relación con aquellos que integran el GC.

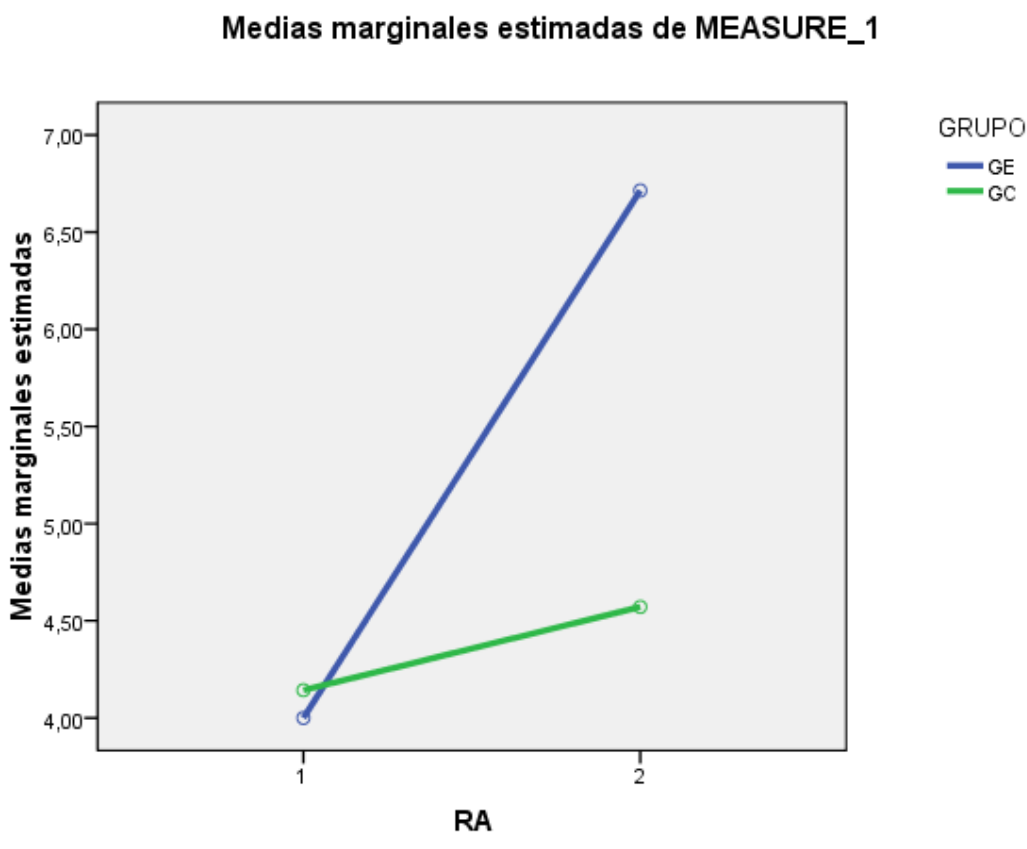

Figura 2. Evolución en la variable RA, según el tipo de grupo

\section{Discusión}

Teniendo en cuenta las limitaciones de este estudio, debido al pequeño número muestral, así como a la influencia de otras posibles covariables, tanto personales, como ambientales (Ben-Itzchak, Abutbul, Bela, Shai \& Zachor, 2016; Zeedyk, Cohen \& Blacher, 2014), que pueden incidir en sus resultados, es posible concluir que el programa de apoyo diseñado, que ha sido aplicado a los integrantes del GE, ha respondido po- 
sitivamente a los objetivos propuestos inicialmente en esta investigación, ya que han permitido facilitar sensiblemente mejoras en el desarrollo de la capacidad de memoria semántica de estos estudiantes, cuyos resultados se diferencian significativamente de aquellos otros obtenidos por los estudiantes que conforman el GC.

Puede deducirse, pues, que la información estructurada, debidamente organizada de acuerdo con los procesos perceptivo-cognitivos, permite a los estudiantes seleccionar y priorizar la información que llega al sistema perceptivo a través de los estímulos externos (aprendizajes). En estos preceptos y en el desarrollo de las relaciones entre los contenidos, ocupa un lugar destacable el conocimiento previamente adquirido en el momento de disponerse a adquirir uno nuevo. El bagaje de conceptos, representaciones y conocimientos previos determinarán el modo en que se organizará la información y el tipo de relaciones que se establecerán entre ellas (Ausubel, Novak \& Hanesian, 1983; Beltrán, Pérez \& Ortega, 2006; Novak, 1982; Novak \& Gowin, 1988), pero, desde las teorías perceptivo-cognitivas, este mecanismo, el cual se produce espontáneamente en personas con un desarrollo normotípico, encuentra, sin embargo, limitaciones severas en las personas con TEA, por lo que es necesario facilitar programas específicos que ayuden en la creación de estas relaciones o nodos entre ambos sistemas perceptivos: conocimiento entrante y conocimiento previo.

En este contexto, para que la secuencia de aprendizaje sea efectiva, es básico que los procesos de codificación cognitiva (que son los facilitadores del paso de una interpretación cognitiva al establecimiento de su representación mental correspondiente), adquieran cierto nivel de significado y coherencia entre ambos niveles perceptivos que operan en el sistema. Pero, además, aun considerando estos principios básicos como elementos esenciales para comprender el funcionamiento psicológico, la eficacia de la estructura didáctica va a depender, asimismo, de las características intrínsecas de los propios contenidos propuestos, así como del nivel de su significatividad (Bruner, 1973).

En este contexto, las teorías cognitivas explicativas, basadas en la Psicología de la Percepción y la Teoría de la Coherencia Central Cognitiva (Happe, 1997; Happe \& Frith, 2006) constituyen las aportaciones empíricas básicas explicativas del funcionamiento del procesamiento perceptivo-cognitivo, las cuales aportan los elementos fundamentales para el diseño de programas específicos. Rutherford y Troje (2012) establecen correlaciones explicativas entre el desarrollo perceptivo-cognitivo del movimiento biológico enmascarado y el cociente intelectual en este colectivo. Woynaroski, Kwakye, Foss-Feig, Stevenson, Stone y Wallace (2013) concluyen, mediante 
estudios basados en la realización de tareas de reconocimiento de sílabas consonantevocales, que existen relaciones entre percepción visual, audiométrica y los tiempos de ejecución. Jaime, McMahon, Davidson, Nevell, Mundy y Henderson (2016) muestran como la atención conjunta, la cual constituye un elemento esencial del proceso perceptivo inicial, se reduce sensiblemente en tareas que exigen coherencia global o atribución de significados en personas con TEA. Freitag et al. (2008) demuestran la existencia de una correlación específica entre la activación neuronal y los procesos de imitación, psicomotricidad gruesa y equilibrio durante tareas de proceso perceptivo de movimientos biológicos sucesivos. Miller (2016) aporta, en este sentido, datos acerca de los programas que fomentan la relación del proceso perceptivo con el desarrollo de las habilidades sociales y el pensamiento crítico, a través del uso de las nuevas tecnologías. Bowker, D’Angelo, Hicks \& Wells (2011) analizan, por su parte, los diferentes programas aplicados, así como la evidencia de sus resultados en personas con TEA, los cuales concluyen que, en efecto, la mayoría de las familias optan por tratamientos multifactoriales para mejorar el desarrollo de sus hijos, así como los programas educativos, basados en las prácticas inclusivas, que inciden en el desarrollo de la percepción de las personas con TEA, siguiendo los principios de las teorías cognitivas (Jones \& Howley, 2010).

El reto educativo consiste, pues, en aplicar las leyes del comportamiento cognitivo al desarrollo específico de los aprendizajes concretos, que no es sino la inclusión de las variables psicológicas en la pedagogía funcional, esto es, el currículo, para lo cual la Psicología de la Percepción constituye, en efecto, un constructo teórico básico a tener en cuenta en el diseño de la construcción de programas psico-socioeducativos facilitadores del desarrollo integrado en personas con TEA (Williams \& Happe, 2009; Williams, Hinshew \& Goldstein, 2015).

En el ámbito científico, son destacables diferentes estudios funcionales que integran tanto los procesos psiconeurobiológicos explicativos del comportamiento (Barton \& Pavilanis, 2012; Bogdashina, 2007; Yeo \& Teng, 2015; Wilson, Dykstra, Watson, Boyd \& Crais, 2012; Young, Rowan, Pardew, Sanford \& Falco, 2009), como aquellos otros componentes sociales y pedagógicos, con el objetivo último de facilitar una respuesta sistémica a todos los elementos explicativos que influyen en una educación de calidad para las personas con TEA (Tonnsen \& Hahn, 2016; Wilson et al., 2012; Yeo \& Teng, 2015; Young et al., 2009); como se desprende de los estudios de Tomasello (1995), estas hipótesis han de aunarse en teorías integradas de carácter funcional. 
Precisamente, en este sentido Ojea (2008b) desarrolla un programa específico precursor de esta nueva investigación, con el objetivo de facilitar el desarrollo de las personas con TEA de nivel 1 que realizan estudios universitarios, cuyo objetivo general es establecer los niveles relacionales o nexos interconceptuales e intercategoriales, entre los nuevos aprendizajes en relación con la información previamente adquirida, cuya estructura general es, en síntesis, la siguiente:

1. Interacción (mediación) social

1.1. Internalización del proceso de mediación social:

1.1.1. Capacidad adquirida: posee la capacidad de mostrar pautas de relación interpersonal.

1.1.2. Capacidad nueva: internalizar el proceso de interacción como proceso de ayuda y solución de problemas.

1.2. Mediadores instrumentales:

1.2.1. Capacidad adquirida: posee la capacidad de solicitar ayuda mediante el proceso de interacción social.

1.2.2. Capacidad nueva: facilitar el uso de mediadores instrumentales.

1.3. Desarrollo autónomo de la mediación.

1.3.1. Capacidad adquirida: comprende el manejo de los mediadores instrumentales.

1.3.2. Capacidad nueva: desarrollar la capacidad para diseñar y organizar el proceso de mediación necesario de forma autónoma.

2. Organización temporal

2.1. Organización del tiempo de estudio:

2.1.1. Capacidad adquirida: posee organización espacio-temporal.

2.1.2. Capacidades nuevas: comprender el manejo de mediadores previsibles de la temporalidad para la organización del trabajo personal.

2.2. Estructuración temporal de forma autónoma:

2.2.1. Capacidad adquirida: comprende el funcionamiento de los mediadores instrumentales de la temporalización de la vida personal.

2.2.2. Capacidad nueva: ser capaz de usar autónomamente los procesos de organización temporal de las actividades personales.

3. Percepción literal: Integración sensorial sucesiva

3.1. Determinar las capacidades adquiridas para la construcción gestáltica (ver apartado 4: Construcción Gestalt). 
4. Construcción gestalt

4.1. Formación de conceptos:

4.1.1. Capacidad adquirida: elabora procesos seriales.

4.1.2. Capacidad nueva: ser capaz de formar unidades conceptuales.

4.2. Generalización en la formación de conceptos:

4.2.1. Capacidad adquirida: elabora procesos sucesivos.

4.2.2. Capacidad nueva: ser capaz de aplicar los aprendizajes realizados en contextos distintos.

4.3. Análisis y síntesis:

4.3.1. Capacidad adquirida: realiza análisis de textos.

4.3.2. Capacidad nueva: ser capaz para deducir ideas coherentes del texto.

4.4.Desarrollo de la coherencia:

4.4.1. Capacidad adquirida: posee la capacidad de realizar análisis básicos.

4.4.2. Capacidad nueva: ser capaz de unir conceptos similares, estableciendo relaciones entre sí.

4.5. Formación de conceptos:

4.5.1. Capacidad adquirida: posee la capacidad de realizar deducciones conceptuales y de establecer relaciones.

4.5.2. Capacidad nueva: ser capaz para deducir ideas básicas a partir de elementos simbólicos: análisis de mapas cognitivos, gráficas, tablas o cuadros sinópticos.

\section{Memoria semántica}

5.1. Elaboración de categorías procedimentales:

5.1.1. Capacidad adquirida: tiene adquirida la capacidad de deducción conceptual, análisis y síntesis.

5.1.2. Capacidad nueva: ser capaz de elaborar categorías procedimentales en base a los conceptos aprendidos.

5.2. Aprendizaje globalizado:

5.2.1. Capacidad adquirida: tiene adquirida la capacidad de elaborar categorías procedimentales.

5.2.2. Capacidad nueva: ser capaz de realizar análisis globales del texto según su significado.

5.3. Recuperación de la información:

5.3.1. Capacidad adquirida: tiene adquirida la capacidad de realizar síntesis globales. 
5.3.2. Capacidad nueva: ser capaz de realizar una recuperación selectiva y coherente de la información a partir de huellas de memoria con significado.

6. Creatividad

6.1. Pensamiento creativo y autónomo:

6.1.1. Capacidad adquirida: posee la capacidad de realizar deducciones conceptuales con significado.

6.1.2. Capacidad nueva: ser capaz de emitir opiniones personales sobre textos.

7. Desarrollo crítico

7.1. Planteamiento de hipótesis

7.1.1. Capacidad adquirida: tiene adquirida la capacidad de realizar opiniones personales.

7.1.2. Capacidad nueva: ser capaz de cuestionar lo aprendido generando nuevas situaciones hipotéticas.

8. Abstracción y simbolismo

8.1. Desarrollo de la ficción:

8.1.1. Capacidad adquirida: tiene adquirida la capacidad de imaginar posibles situaciones distintas a las originales.

8.1.2. Capacidad nueva: ser capaz de elaborar respuestas imaginadas a dichas situaciones hipotéticas.

8.2. Resolución de problemas:

8.2.1. Capacidad adquirida: tiene adquirida la capacidad de crear una ficción.

8.2.2. Capacidad nueva: ser capaz de estructurar procesos de solución de problemas concretos.

\section{Lista de referencias}

American Psychiatric Association. (2013). Manual diagnóstico y estadístico de los trastornos mentales DSM-5. Madrid: Panamericana.

Ausubel, D. P., Novak, J. D., \& Hanesian, H. (1983). Psicología educativa: un punto de vista cognoscitivo. México: Trillas.

Barton, E. E., \& Pavilanis, R. (2012). Teaching pretend play to young children with 
autism. Young Exceptional Children, 15(1), 5-17.

Belinchón, M., Rivière, A., \& Igoa, J. M. (1992). Psicología del lenguaje. Investigación y teoría. Madrid: Trotta.

Beltrán, J. A., Pérez, L. F., \& Ortega, M. I. (2006). CEA Cuestionario de Estrategias de Aprendizaje. Madrid: TEA.

Ben-Itzchak, E., Abutbul, S., Bela, H., Shai, T., \& Zachor, D. A. (2016). Understanding one's own emotions in cognitively-able preadolescents with autism spectrum disorder. Journal of Autism and Developmental Disorders, 46(7), 2363-2371.

Bogdashina, O. (2007). Percepción sensorial en el autismo y síndrome de Asperger. Experiencias sensoriales diferentes, mundos perceptivos diferentes. España: Autismo Ávila.

Bölte, S., Holtman, M., Poustka, F., Scheurich, A., \& Schmidt, L. (2007). Gestalt perception and local-global processing in high-functioning autism. Journal of Autism and Developmental Disorders, 37(8), 1493-1504.

Bowker, A., D’Angelo, N. M., Hicks, R., \& Wells, K. (2011). Treatments for autism: parental choices and perceptions of change. Journal of Autism and Developmental Disorders, 41(10), 1373-1382.

Bruner, J. S. (1973). Beyond the information given: Studies in the psychology of knowing. Nueva York: Norton.

Dretske, F. I. (1981). Knowledge and the flow of information. Cambridge: MIT Press.

Evers, K., de Wit, L., van der Hallen, R., Haesen, B., Steyaert, J., Noens, I., \& Wagemans, J. (2014). Brief report: reduced grouping interference in children with ASD. Evidence from a multiple object tracking task. Journal of Autism and Developmental Disorders, 44(7), 1779-1787.

Fodor, J. A: (1983). The modularity of mind. Cambridge, Massachusetts: MIT Press.

Freitag, C. M., Konrad, C., Haberlen, M., Kleser, C., von Gontard, A., Reith, W., Troje, N. F., \& Krick, C. (2008). Perception of biological motion in autism spectrum disorders. Neuropsychologia, 46(5), 1480-1494.

Frith, U. (2004). Autismo. Hacia una explicación del enigma ( $2^{\mathrm{a}} \mathrm{ed}$.). Madrid: Alianza. Gardner, H. (1985). The mind's new science. Nueva York: Basic Books.

Gibson, J. J. (1979). The ecological approach to visual perception. Boston: Houghton Mifflin.

Hamilton, A. F., Brindley, R., \& Frith, U. (2009). Visual perspective taking impairment in children with autistic spectrum disorder. Cognition, 113(1), 37-44.

Happe, F. (1997). Central coherence and theory of mind: reading homographs in context. British Journal of Development Psychology, 15, 1-12. 
Happe, F., \& Frith, U. (2006). The weak coherence account: detail focused cognitive style in autism spectrum disorders. Journal of Autism and Developmental Disorders, $36(1), 5-25$.

Iarocci, G., Rombough, A., Yager, J., Weeks, D. J., \& Chua, R. (2010). Visual influences on speech perception in children with autism. Autism: The International Journal of Research and Practice, 14(4), 3015-320.

Jaime, M., McMahon, C. M., Davidson, B. C., Nevell, L. C., Mundy, P. C., \& Henderson, H. A. (2016). Brief report: reduced temporal central EEG alpha coherence during joint attention perception in adolescents with autism spectrum disorder. Journal of Autism and Developmental Disorders, 46(4), 1477-1489.

Johnson-Laird, P. N. (1983). Mental models. Towards a cognitive science on language, inference and conciousness. Cambridge: Harward University Press.

Jones, K., \& Howley, M. (2010). An investigation into an interaction programme for children on the autism spectrum: outcomes for children, perceptions of schools and a model for training. Journal of Research in Special Educational Needs, $10(2), 115^{-123 .}$

Liu, Y., Cherkassky, V. L., Minshew, N. J., \& Just, M. A. (2011). Autonomy of lower level perception from global processing in autism: Evidence from brain activation and functional connectivity. Neuropsychologia, 49(7), 2105-2111.

Maister, L., \& Plaisted-Grant, K. C. (2011). Time perception and its relationship to memory in autism spectrum conditions. Developmental Science, 14(6), 13111322.

Miller, D. J. (2016). Educator perceptions of visual support systems and social skills for young adults with autism spectrum disorders. Washington: Walden University/ ProQuest LLC/D. Dissertation.

Novak, J. D. (1982). Teoría y práctica de la educación. Madrid: Alianza.

Novak, J. D., \& Gowin, D. B. (1988). Aprendiendo a aprender. Barcelona: Martínez Roca.

Ojea, M. (2006). Síndrome de Asperger: teoría de la Coherencia Central Cognitiva. Implicaciones didácticas y organizativas. Ponencia presentada en las Jornadas sobre Síndrome de Asperger y Educación, de la Asociación Gallega de Familias de Personas afectadas por el Síndrome de Asperger, Fundación María José Jove, La Coruña, España.

Ojea, M. (2008a). Desarrollo perceptivo e integración cognoscitiva en estudiantes con síndrome de Asperger en el ámbito universitario: un estudio de caso. En 
M. Belinchón (Ed.), I Encuentro Nacional de Investigadores de los Trastornos del Espectro Autista (TEA) (pp. 415-425). Madrid: Universidad Autónoma de Madrid.

Ojea, M. (2008b). Síndrome de Asperger en la Universidad: percepción y construcción del conocimiento. Alicante: Club Universitario.

Pylyshyn, Z. (1984). Computation and cognition. Cambridge: MIт Press.

Rondan, C., \& Deruelle, C. (2007). Global and configural visual processing in adults with autism and Asperger's Syndrome. Research in Developmental Disabilities: A Multidisciplinary Journal, 28(2), 197-206.

Rumelhart, D. E., \& McClelland, J. L. (1986). Parallel distributed processing. Massachusetts: MIT Press.

Rutherford, M. D., \& Troje, N. F. (2012). IQ Predicts biological. Motion perception in autism spectrum disorder. Journal of Autism and Developmental Disorders, $42(4), 557-565$.

Sheppard, E., Ropar, D., Underwood, G., \& van Loon, E. (2010). Brief report: driving hazard perception in autism. Journal of Autism and Developmental Disorders, 40(4), 504-508. doi:10.1007/s10803-009-0890-5

Solières, I., Mottron, L. Saumier, D., \& Larochelle, S. (2007). Atypical categorical perception in autism: autonomy of discrimination? Journal of Autism and Developmental Disorders, 37(3), 481-49o.

Tomasello, M. (1995). Understanding the self as a social Agent. En P. Rochat (Ed.), The self in infancy: theory and research (pp. 449-46o). Amsterdam: North Holland/Elsevier Science.

Tonnsen, B. L., \& Hahn, E. R. (2016). Middle School Students' Attitudes toward a Peer with Autism Spectrum Disorder: Effects of Social Acceptance and Physical Inclusion. Focus on Autism and Other Developmental Disabilities, 31 (4), 262274. Recuperado de http://dx.doi.org/10.1177/1088357614559213

Vygotsky, L. S. (1972). Pensamiento y lenguaje. Buenos Aires: La Pléyade.

Vygotsky, L. S. (1979). El desarrollo de los procesos psicológicos superiores. Barcelona: Crítica.

Walsh, J. A., Creighton, S. E., \& Rutherford, M. D. (2016). Emotion perception or social cognitive complexity: what drives face processing deficits. Journal of Autism and Developmental Disorders, 46(2), 615-623.

Williams, D. L., Hinshew, N. J., \& Goldstein, G. (2015). Further understanding of complex information processing in verbak adolescents and adults with autism 
spectrum disorders. The International Journal of Research and Practice, 19(7), 859-867.

Williams, D. M., \& Happe, F. (2009). Preconceptual aspects of self-awareness in autism spectrum disorder: the case of action monitoring. Journal of Autism and Developmental Disorders, 39, 251-259.

Wilson, K. P., Dykstra, J. R., Watson, L. R., Boyd, B. A., \& Crais, E. R. (2012). Coaching in early education classrooms serving children with autism: A pilot study. Early Childhood Education Journal, 4o(2), 97-105.

Woynaroski, T. G., Kwakye, L. D., Foss-Feig, J. H., Stevenson, R. A., Stone, W. L., \& Wallace, M. T. (2013). Multisensory speech perception in children with autism spectrum disorders. Journal of Autism and Developmental Disorders, 43(12), 2891-2902.

Yamasaki, T., Fujita, T., Ogata, K., Goto, Y., Munetsuna, S., Kamio, Y., \& Tobimatsu, S. (2011). Electrophysiological evidence for selective impairments of optic flow perception in autism spectrum disorder. Research in Autism Spectrum Disorders, 5(1), 400-407.

Yeo, K. J., \& Teng, K. Y. (2015). Social skills deficits in autism: a study among students with autism spectrum disorder in inclusive classrooms. Universal Journal of Educational Research, 3(12), 1001-1007.

Young, H., Rowan, J., Pardew, M., Sanford, T., \& Falco, R. (2009). Autism partnership program: Parents \& educators partnering to improve outcomes for children and youth with autism [manuscrito no publicado]. Portland: Portland State University.

Yuste, C. (2005). MY Tests de Memoria. Madrid: TEA.

Zeedyk, S., Cohen, S. R., \& Blacher, J. (2014). Syndrome specific impact on parental wellbeing: autism compared. En V. Preedy (Ed.), The comprehensive guide to autism (pp. 625-650). Nueva York: Springer. 


\section{ANEXOS}

\section{Anexo 1: Test my de Memoria Semántica, nivel III, de Yuste (2005) (texto, cuadernillo, hoja de respuestas, plantilla).}

Se recogen las Hojas de respuestas, lápices y gomas y se da por finalizada la aplicación.

\subsection{Instrucciones específicas para el nivel III}

Una vez sentados separadamente los sujetos y motivados para el examen, se reparten los lápices, gomas y Hojas de respuestas y se les pide que anoten los datos de identificación en la parte superior. A continuación se llama su atención y se dice:

"Estad atentos, porque voy a leeros una redacción bastante larga, para que luego contestéis a unas preguntas acerca de ella. No hace falta que intentéis recordar todos los datos que se lean y menos en el mismo orden. Ahora escuchad atentamente.

(CON VOZ PAUSADA Y CLARA SE LEE LA HISTORIA UNA SOLA VEZ)

\section{La conquista del espacio}

La Luna ya no constituye un enigma insalvable para la curiosidad del hombre, pues entre los años 1969 y 1972 fueron varios los astronautas que pisaron el enigmático astro, buscando desciffrar sus incognitas. 
Después del primer descenso del hombre en la Luna, se sucedieron los viajes tripulados. En el segundo viaje, a bordo del módulo hunar Intrepid, dos astronautas norteamericanos descendieron en el Océano de las Tempestades, instalaron un conjunto de aparatos científicos mucho más complejos que en el descenso anterior y recorrieron a pie un par de kilómetros. Terminada la misión y después de despegar, la cabina del módulo, ya inútil, fue despedida y proyectada contra la superficie lunar a fin de crear un pequeño temblor de tierra para probar el funcionamiento del sismómetro instalado. La prueba proporcionó la gran sorpresa de que las ondas detectadas mantuvieron su vibración más de una hora, como si la Luna fuese una gigantesca caja de resonancia.

El quinto viaje se realizó sin ninguna incidencia digna de ser notada. El módulo Ituar Falcon tomó tierra en el sitio previsto, y los astronautas efectuaron tres salidas de reconocimiento, empleando en ellas un pequeño automóvil plegable, el Rover, que en adelante seria tun imprescindible instrumento de exploración. Recogieron 80 kilos de muestras lunares, visitaron algunos cráteres y en total recorrieron unos 26 killometros sin alejarse mucho de la nave.

El proyecto Apolo, nombre dado a la serie de lanzamientos lunares, terminó con el lanzamiento del Apolo-17. Ceman fue, por el momento, el áltimo ser humano en pisar el suelo lunar. Dejo adosada a tua pata del tren de aterrizaje la siguiente inscripción:

'Aqui terminó el hombre sus primeras exploraciones de la Luna. Diciembre de 1972. Que el espiritu de paz con que vinimos se refleje en las vidas de toda la humanidad:

La Unión Soviética habla renunciado a enviar naves tripuladas a la Luna, pero no a explorarla con medios mecánicos. Asi, logró emiar varias naves con taladros, brazos mecánicos para recoger muestras y equipo de laboratorio para realizar los análisis de las muestras recogidas. Los soviéticos llegaron a enviar a la Luna tun pequeño vehículo con ruedas que recorrió más de diez kilómetros en el transcur. so de los once meses que permaneció activo.

Después de conquistada la Luna, los esfuerzos se dirigieron hacia la construcción de estaciones espaciales a las que pudiesen atracar las naves tripuladas en sus desplazamientos por el universo. De esta manera nacieron la Salyut por parte de Rusia y el Skylab de Estados Unidos, a las que lograron atracar numerosas naves tripuladas y batir los récords de permanencia en el espacio.

Venus también ha sido objeto de exploraciones con naves espaciales sin tripulantes. Se ha llegado a saber por medio de las fotografías obtenidas que su atmósfera es 60 veces más densa que la de la Tierra y la temperatura de más de $400 \mathrm{gra}$. dos centigrados. El aire apenas tiene oxigeno y si un 97 por ciento de anhidrido carbónico.

Marte nos va desvelando su imagen mítica. Ya se sabe que su atmósfera y superficie son muy distintas a las de la Tierra, que no tiene apenas cráteres, que su atmós. fera es casi imperceptible y que su temperatura oscila entre los 15 y los 150 gra. 
Desarrollo de la capacidad de memoria semántica a

través de la formación de redes relacionadas

Manuel Ojea Rúa

C. Yusre Heavir

dos bajo cero. Los dos satelites de Marte, Deimos y Febos, parecen periascos de foma irregular y con múltiples cráteres formados por el impacto de los meteoritos a lo largo de los milenios.

Y el hombre sigue adentrándose en los misterios del universo, enviando naves prác. ticamente a todo el sistema solar, incluryendo Júpiter, Satumo y Mercurio, y procurando descifrar los enomes enigmas que siempre planteará el universo, pues aún se conoce mury poco o casi nada de las estrellas, las galaxias y los limites del uni. verso en el que las distancias se miden por miles de millones de años luz".

A continuación, pidiéndoles silencio y que no se comuniquen entre sí, se reparten los Cuadernillos de este Nivel III, dejándolos con la portada hacia arriba. Luego, y como entrenamiento, el cxaminador resuelve en voz alta los dos ejemplos existentes en la portada del Cuademillo, pidiendo a los sujetos que contesten en sus Hojas de respuestas. Cuando todos hayan comprendido la tarea a realizar y el modo de anotar sus respuestas (es preferible que señalen poniendo una cruz, para que se visible a través de la plantilla de corrección), se dice:

"Vais a contestar a las preguentas existentes en el Cuadernillo como lo habeis hecho en los ejemplos de entrenamiento. No hagáis ninguna señal en el Cuadernillo; anotad todas vuestras respuestas en la Hoja. Trabajad con onden y siguiendo la numeración de las cuestiones; si no os acordais de un dato para contestar, dejadla en blanco y pasad a la siguiente cuestión y si tenéis tiempo, al final podéis volver a ella. No os entretengáis demasiado en una pregunta. ¿Habéis com. prendido?... "Comenzad!"

En este momento se pone cl cronometro en marcha y se conceden:

-15 minutos para $1^{\circ}$ de ESO.

- 12 minutos para $2^{\circ}$ de ESO en adelante.

No obstante, no se debe forzar la marcha de los sujetos con deficiente capacidad lectora. Si pasado el tiempo concedido todavía estuviera trabajando una buena parte del grupo de sujetos, se puede ampliar prudencialmente el tiempo indicado.

Al terminar, se recoge el material (Hojas, lápices y gomas) y se da por finalizada la aplicación.

\subsection{Corrección y obtención de puntuaciones directas}

Las normas son específicas para cada Nivel y las puntuaciones directas se obtienen con la ayuda de la correspondiente plantilla de correceión.

\section{Nivel Elemental}

En general, se concede un punto por cada elemento acertado y se resta un punto por cada respuesta incorrecta; la puntuación máxima es de 44 puntos. 
Manuel Ojea Rúa

N.० 108

\title{
MEMORIA III
}

\author{
CUADERNILLO
}

\begin{abstract}
En este impreso encontrará una serie de frases o preguntas relacionadas con el texto que acaba de lecr el examinador. A continuación se presentan seis alternativas de respuesta y sollo una de ellas con. testa a la pregunta o completa la frase propuesta.

Lea el contenido de ta frase y las seis alternativas de rospuesta para ver cubl es la acertada; fijese en la letra que lleva delante (A a F) y marque la contestaclón correcta en la Hoja de respuestas, en is linea que corresponda.

Siga el orden de numeración de las frases y asi evitará errores en las anotacionos.

Para hacer una pequeña práctiça. leo los dos ejemplos que vionen aquí debajo y conteste en la Hoja do respuestas.
\end{abstract}

Ejemplo 1: ¿Cuall era el título de la redacción leida?
A. El espacio
B. Los vuelos espaciales tripulados
C. La conquista del universo
D. El Sistema Solar
E. La conquista del espacio
F. La era espacial

Ejemplo 2: La luna ya no constituye...

A. Un misterio

B. Un enlgma insalvable para la curiosidad del hombre

C. Ninguna novedad para las actuales goneraciones

D. El antiguo deseo de explorarla

E. La antigua novedad por explorarla y conocerla

F. Un problema insoluble para la mente humana.

Las respuestas acertadas son: E, en el ejemplo 1, y B, en el 2. minacion.

Trabaje con rapidez, pero procure no cometer errores, y deténgase cuando se de la sefial de ter.

AHORA, ESPERE, NO PASE A LA PAGINA SIGUIENTE HASTA QUE SE LE INDIOUE

julio-diciembre, 2017 | ISSN 1870-5308

Instituto de Investigaciones en Educación | Universidad Veracruzana 
Manuel Ojea Rúa

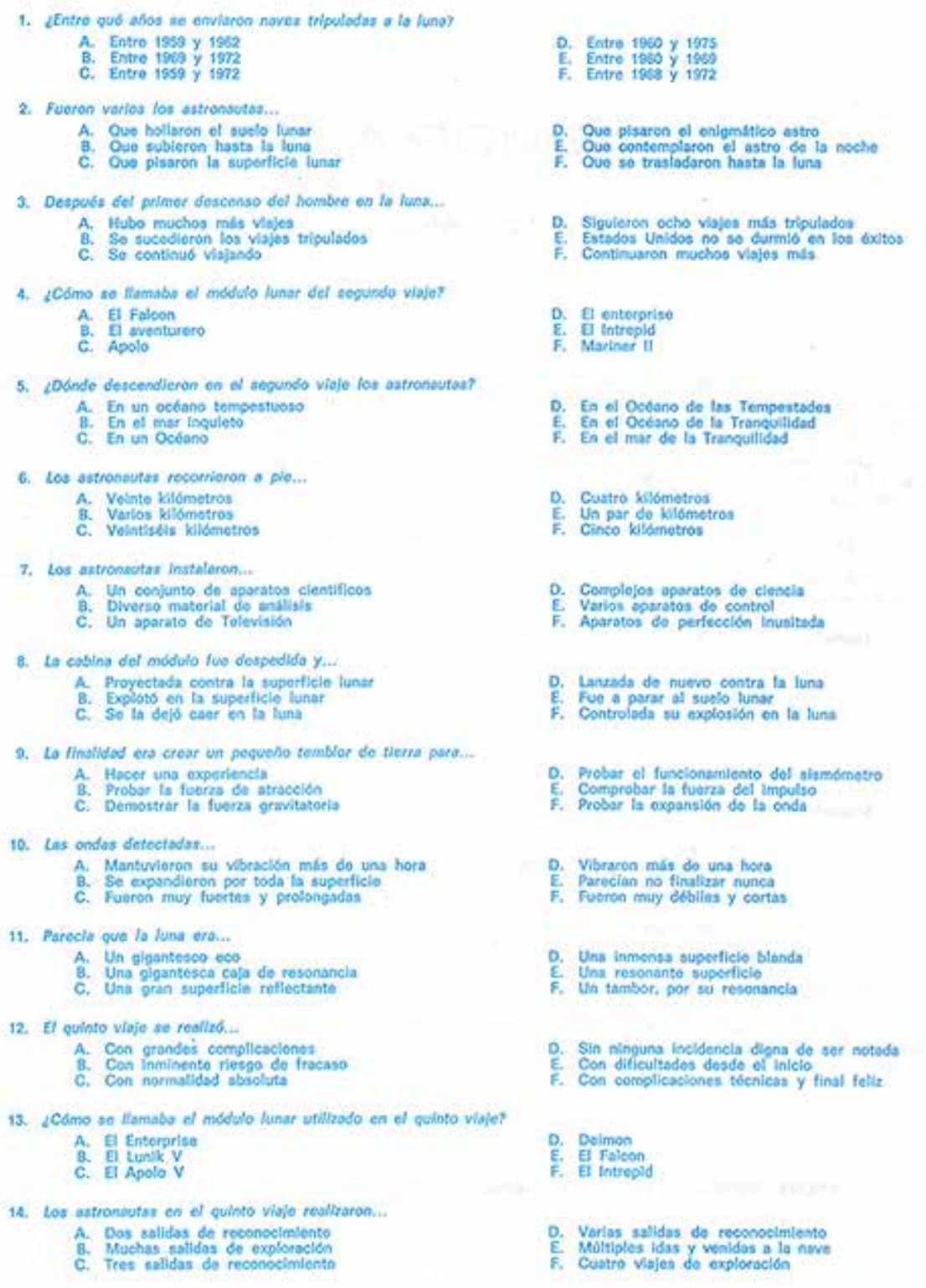


Manuel Ojea Rúa

15. Emplearon en tos safides efectuades...
A. Una casa rodante
c. Un poquento suto, of flover
D. Un automovil con tres ruedss, el Rover
F. Un pequotio astomovil plogable, ol Rover

16. En sdelante seria..

A. Un imprescindibla fastrumento do exploración

B. Un lastrumbito necesario en los salldas

D. Muy ótil en las exploraclones

F. Lacosutrio so impresclindible

17. En el çulinto walo rocogieron...

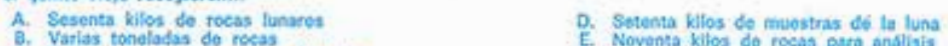

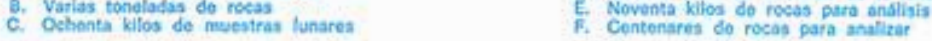

16. Visitaron tomblón...

A. Tres o ewatro cratores

C. Muchos critero:

O. Eastantes ensteres

F. Cinco cráterea

19. En totot rocorrieron sin stojarso mucho de to nowe...

A. Unos 20 kilometros

B. Unos 36 vilometros

D. Unos 25 kilomotron

F. Unos to kilomotros

20. El proyecto Apoto termins con of tanzamitento...

A. Dot Apois Diocisols

c. Dol Apos Dlocisiete
c. Det intropid

D. Del Survoyor
E. Del Apolo Dieclocho

F. Del Apolo Doce.

21. El bittimo ser humano en pisor ef suelo funst fuo, por el nomento.

$\begin{array}{ll}\text { A. Cobstan } & \text { D. Fordinand } \\ \text { B. Collins } & \text { E. Conred }\end{array}$

C. Williams

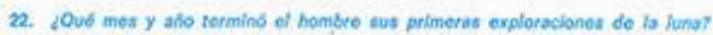

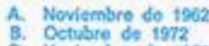
c. Novientiono de 1972
D. Diclamber do 1973
F. Soptlombre de 1 ing

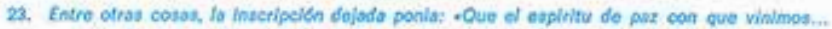

A. Sirva paza la paz de tods is hemanidad

B. Se refiejo en las vidas do toda la humanidad

D. Contribuya of mantecimiento do lo paz on la tientra

E. So mantomos a lo lareo do todos los sigloa

24. La UnlGa Sowtelea no habla renunciado e explorar ta luna.

A. Con medios mechinieos
B. Coo los medios a su nleance
C. Con medios propios

D. Con naves tripulados

loant creviar varias noves con.

25. Buata logró enviar varias noves ean..

A. Tripulantes a bores

C. Talidros, beazos meesnicos

E. Con sistemas proplos mecasizados
F. Hasts conseguir fosoyjafialla completamente

26. Los equipos do laboraterio eran.

A. Para hacer exporimentos
B. Para fotopraftar ta luna
C. Para tolevisar la llogada

A. Para hocer experimentos
B. Para fotograflar to luna
C. Para tolevisar la llogada

D. Oycimss lastrumentos cleotsficos

E. Gistemas mecanizedon may perfectos

27. Los seriteicos llegaroa a emviar a ta funs..

A. Un vehículo con tres ruodas

B. Un auto plegable, el Rover

D. Para realizer el anstists do las musstras

F. Para reanalizar pruobas do solsmometria

c. Un autornowit mary masejablo

D. Uo pequefio whiculo con ruedas

E. Un oxtrinho vebleuto gli nuedas
F. Un olgantesco vehiculo con ruedas

julio-diciembre, 2017 | ISSN 1870-5308

Instituto de Investigaciones en Educación | Universidad Veracruzana 
28. Dospuda do conguistada la funo los eatueraos se difigleron...

A. A otros sotelites
c. A descifras otros enigmas
C. A recopliar nuevos dstos

D. A otros planetas más alojados

E. Hacla la conatruceín do estaciones espactales

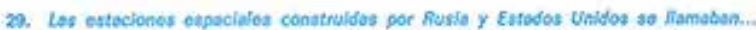

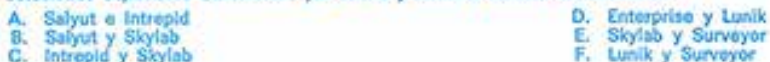

30. Con elles lograron butio...

A. Los limites de permanencia en of espateio

a. Los reoords do resistenela on estodo do ingrividez

D. Muchas marcas espaclates do resilstencia

E. Tesss las ospotativs do estancia en ol espocio

31. En comparación con to tierrs lo atmositera do Venus es....

A. Veinte voces mils densa

E. tofinitasnonte más deasa
c. Sesonta veces mis densa

D. Mueho más donsa

E. Mucho mas pesada y donsa

32. La tomporatura de Venus on.

A. Do 400 grados ceotigrodos

C. De más de 400 grados centigrados

D. Do 400 grados bajo cero

E. Do mas do s.o grados acbien cero

33. El atre de Venus contiane.

A. Gran cantided so exigeno

C. Un tres por clento to Oxjigum

D. Un 97 por clento do Oxigeno

E. Un 87 por clento do Nitrópeno irregalirablo

34. Respecto a la atmosfera de Marte podemos docir.t.

A. Es procticamente inexistente

8. Su atmolafera es casil irnoorceptible
C. Es enśs donsa quo ta de ta tlema

D. No existe atmolera

E. No es oponas porceptiole
F. Es 00 veces mis densa pao ta tierra

35. Su temporatura otcilla entre...

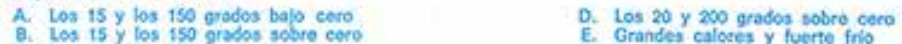

$\begin{array}{ll}\text { C. Los } 25 \text { y los } 250 \text { grodos bojo cero } & \text { E. Grandes calores y luerto frio } \\ \text { C. Los } 25 \text { y } 250 \text { grados sobre cero }\end{array}$

28. ¿Como to llaman los dos satelitos do Marte?

A. Argos y Fobos
B. Delnios y Aross

C. Deinos y Argos

D. Interapos y Fobos

E. Fobos : Intreopld

37. Los dos sutdised do Marto parecen.
A. Dos plodras de forms rogular
c. Pehaseos do forma regular
D. Paflascos de forma irregulyt
E. Pledras con mulehis itrecularidodesteres

38. Tienen mütuples criteres formidos por el impocto de tos meteoritas...

A. A lo largo de los siglos

C. A lo largo do los milenios

D. A lo largo do los ahos

C. En millones do afios

F. A to largo do ta tistocis

39. So han enviodo noves prefticamonte a todo of siatema solev, incicyendo...

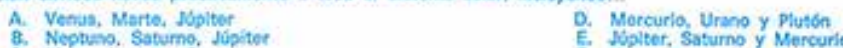

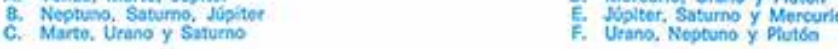

40. Lat ditatancias en el timverso ac miden poe...
A. Milles de millones do axios fur
c. Anos tou
D. Mittones de antos luz
F. Centenaros do atios liur 
N.108

MEMORIA - III

Hojo do rospuestas

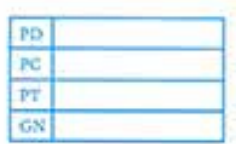

Sexo

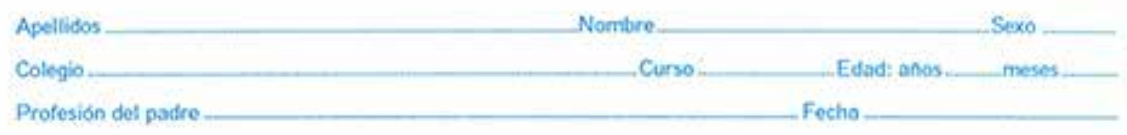

Profesión del padre -

SI DESEA CAMBIAR UNA CONTESTACION.

BORRE BIEN LA EQUIVOCADA Y MARQUE LA NUEVA RESPUESTA

Ejemplos

1. A B C D E F

2. A B C D E F

\begin{tabular}{|c|c|c|c|c|c|c|c|c|c|c|c|c|c|}
\hline 1. & A & B & C & D & E & F & 21. & A & B & C & D & E & $\mathrm{F}$ \\
\hline 2. & A & B & c & D & E & F & 22. & A & B & C & D & $\mathrm{E}$ & F \\
\hline 3. & A & B & C & D & E & F & 23. & A & B & C & D & E & F \\
\hline 4. & A & B & C & D & E & $F$ & 24. & A & B & c & D & E & F \\
\hline 5. & A & B & C & D & E & F & 25. & A & B & C & D & $\mathbf{E}$ & F \\
\hline 6. & A & B & C & D & E & $F$ & 26. & A & B & C & D & E & F \\
\hline 7. & A & B & C & D & $\mathrm{E}$ & F & 27. & A & B & C & D & E & $\mathrm{F}$ \\
\hline 8. & A & B & C & D & E & F & 28. & A & B & C & D & $\mathbf{E}$ & $\mathrm{F}$ \\
\hline 9. & A & B & c & D & $\mathrm{E}$ & F & 29. & A & B & c & D & $\mathbf{E}$ & $F$ \\
\hline 10. & A & B & c & D & E & $F$ & 30. & A & B & C & D & E & F \\
\hline 11. & A & B & c & D & E & $F$ & 31. & A & B & C & D & $\mathbf{E}$ & F \\
\hline 12. & A & B & C & D & E & $F$ & 32. & $A$ & B & C & D & $\mathbf{E}$ & F \\
\hline 13. & A & B & C & D & $\hat{E}$ & $\boldsymbol{F}$ & 33. & A & B & C & D & E & $F$ \\
\hline 14. & A & B & C & D & E & $\mathrm{F}$ & 34. & A & B & C & D & $\mathrm{E}$ & F \\
\hline 15. & A & B & C & $\mathrm{D}$ & $\mathrm{E}$ & $\mathrm{F}$ & 35. & A & B & C & D & E & F \\
\hline 16. & A & B & C & D & E & F & 36. & A & B & C & D & E & F \\
\hline 17. & A & B & C & D & E & $F$ & 37. & A & B & C & D & $\mathrm{E}$ & F \\
\hline 18. & A & B & C & D & $\mathbf{E}$ & $\mathrm{F}$ & 38. & A & B & C & D & E & $\mathrm{F}$ \\
\hline 19. & A & B & c & $\mathrm{D}^{*}$ & E & $F$ & 39. & A & B & C & D & E & $\mathrm{F}$ \\
\hline 20. & $A$ & B & C & D & E & $F$ & 40. & A & B & C & D & E & $\mathrm{F}$ \\
\hline
\end{tabular}




\title{
MEMORIA - Nivel III
}

\section{Plantilla}

\author{
$(P D=$ Aciertos; PD máx. -40$)$
}

CONFIOENCIAL: Esta plantilla contiene la clave de la prueba. Guárdela de forma que no sea visto por otras personas.

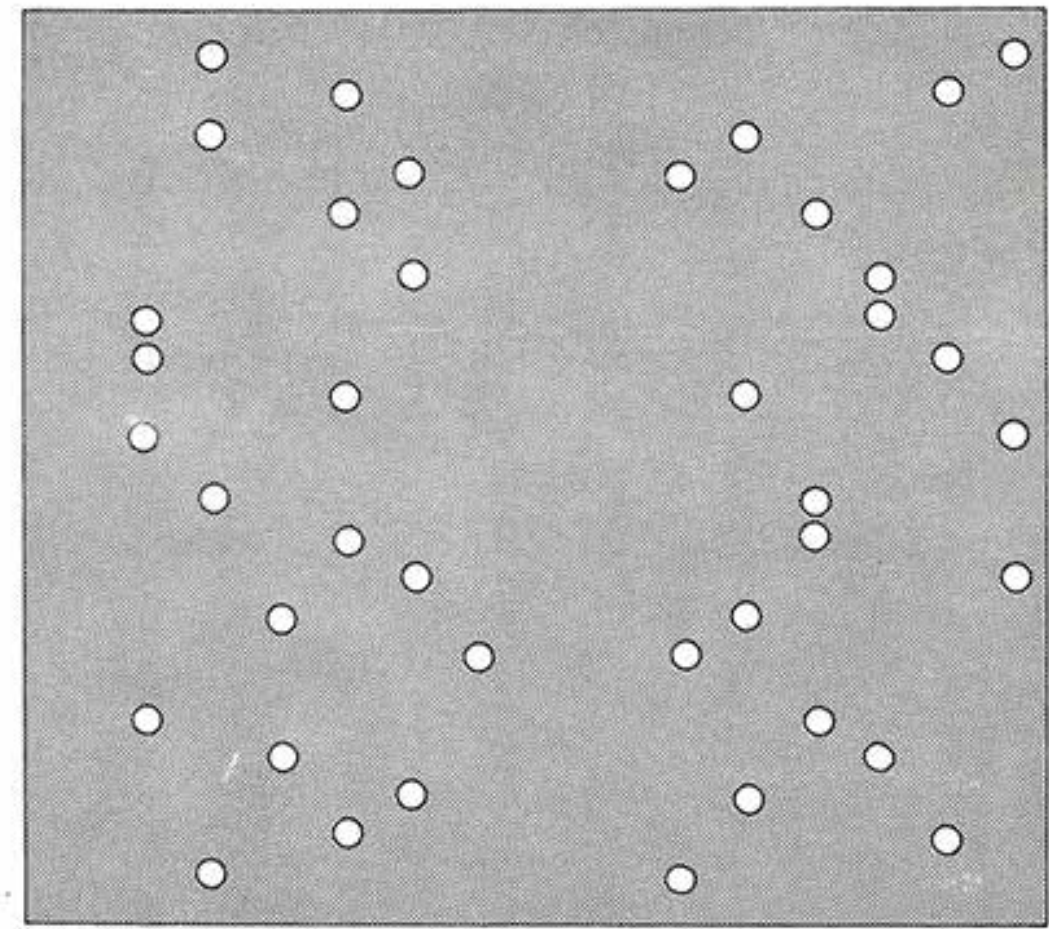

